\title{
Pobreza intergeracional no complexo de favelas do São João - Rio de Janeiro - Brasil ${ }^{1}$

\author{
Intergenerational poverty in the slum complex of the São João - Rio \\ de Janeiro - Brazil
}

\author{
Dra. Astrid Maciel Motta, Instituto Educacional Almenara - ALFA \\ astridmotta@hotmail.com | https://orcid.org/0000-0003-0041-2825 \\ Calle Mario José de Souza 11, Parque São João. Almenara, Minas Gerais. Brasil. \\ Dra. Cristina Ribeiro Parente, Universidade do Porto \\ cparente@letras.up.pt | http://orcid.org/0000-0002-7500-7050 \\ Via Panorâmica s/n, Porto, Portugal
}

\begin{abstract}
Resumo
Neste artigo discute-se uma abordagem multidimensional sobre a pobreza, destacando variáveis que permitem aferir e interpretar a reprodução, ou não, da pobreza intergeracional (Bird, 2007). A investigação de cariz exploratório e descritiva privilegiou uma metodologia qualitativa baseada em entrevistas semiestruturadas, realizadas com 29 moradoras e moradores do complexo de favelas do São João, no Engenho Novo, Rio de Janeiro. Adotamos um padrão de análise comparativa entre três gerações: a primeira geração (G1) é dos progenitores; a segunda, a geração em análise (G2); seus descendentes são a G3; a análise alicerçou-se num raciocínio sistemático de comparação de 23 variáveis para criar índices compósitos de classificação das famílias. O estudo apontou que as dificuldades enfrentadas por moradoras e moradores das favelas do Rio de Janeiro desencadeiam mecanismos produtores e transmissores da pobreza e que os membros das famílias, mesmo tendo acesso à educação e a algumas medidas de política pública, mantiveram-se maioritariamente em condição de pobreza, dadas as dinâmicas sociais vivenciadas no contexto da favela. Os fatores promotores do ciclo intergeracional da pobreza nestes territórios estão presentes na dimensão intra e extrafamiliar, conforme nos remete Bird (2007). Deste modo, somos a concluir que a efetividade das políticas públicas destinadas ao rompimento da pobreza exigem estudos aprofundados sobre as dinâmicas sociais específicas ao contexto das favelas cariocas para subsidiar a formulação de medidas consubstanciadas nas particularidades do local.
\end{abstract}




\begin{abstract}
This paper discusses a multidimensional approach to poverty, highlighting variables that allow us to gauge and interpret the reproduction of intergenerational poverty (Bird, 2007). This exploratory and descriptive research privileged a qualitative methodology based on semi - structured interviews conducted with 29 residents of the São João favelas complex, in Engenho Novo, Rio de Janeiro. We adopted a standard of comparative analysis between three generations: first generation are the progenitors (G1); the second, the generation under analysis (G2); and their descendants are (G3); the analysis was based on a systematic reasoning of comparison of 23 variables to create composite classification indices of the families. The study pointed out that the difficulties faced by residents of the favelas of Rio de Janeiro trigger mechanisms that produce and transmit poverty, and that family members, even though they had access to education and some public policy measures, remained largely in poverty, given the social dynamics experienced in the context of the favela. The factors promoting the intergenerational poverty cycle in these territories are present in the intra-and extra familial dimension, as referred to by Bird (2007). In this way, we conclude that the effectiveness of public policies aimed at the disruption of poverty require in-depth studies on the social dynamics specific to the context of the Rio de Janeiro favelas to subsidize the formulation of measures consubstantiated in the particularities of the place.
\end{abstract}

Palavras-chave: pobreza, Reprodução, Gerações, Ciclo intergeracional da pobreza.

Key words: poverty, Reproduction, Generations, Intergenerational cycle of poverty.

\title{
1. INTRODUÇÃO
}

Todo ser humano tem direito a um padrão de vida capaz de assegurar a si e à sua família saúde e bem-estar, inclusive alimentação, vestuário, habitação, assistência médica e os serviços sociais indispensáveis e direito à segurança em caso de desemprego, doença, invalidez, viuvez, velhice ou outros casos de perda dos meios de subsistência em circunstâncias fora de seu controle? ${ }^{2}$.

(Declaração Universal dos Direitos Humanos, Art. 25, 1, adotada e proclamada pela resolução 217 A, III, da Assembleia Geral das Nações Unidas, em 10 de dezembro de 1948).

A pobreza é um processo complexo e está relacionado a algum tipo de privação constituída por elementos de ordem material, não material, cultural e social na vida de uma pessoa ou família. Como categoria sociológica, encontramos formulações diversas para definir pobreza e suas causas; para subsidiar a formulação de políticas públicas destinadas ao enfrentamento da pobreza, ela é traduzida em dados estatísticos pelos quais se estabelecem parâmetros para definir oficialmente o que é pobreza ou extrema pobreza. Pensadores como Raczynski (2002) concebem a pobreza nas dimensões materiais e não materiais, ao considerar elementos menos tangíveis, 
entre eles aspectos atitudinais, condutas e valores. Para Hagenaars e De Vos (1988), todas as definições de pobreza podem ser enquadradas em uma destas três categorias: a) pobreza é ter menos do que um mínimo objetivamente definido (pobreza absoluta); b) pobreza é ter menos do que outros na sociedade (pobreza relativa); c) pobreza é sentir que não tem o suficiente para seguir adiante (pobreza subjetiva). Entre os mais variados conceitos, definições e debates sobre pobreza, podemos destacar as contribuições de Amartya Sen no início dos anos 1980. Segundo ele, uma característica da pobreza é que ela possui "irredutível essência absoluta": um de seus elementos óbvios são a fome e a inanição; não importa qual seja a posição relativa na escala social, aí certamente existe pobreza. O fato de algumas pessoas terem padrão de vida mais baixo que outras é certamente uma prova de desigualdade, mas não pode, por si só, ser prova de pobreza, a menos que saibamos mais a respeito da qualidade de vida que essas pessoas de fato acessam (Sen, 2000).

Neste estudo adotamos conceitualmente que,

a pobreza é um fenômeno multidimensional em que há a falta do que é necessário para o bem-estar material. Associa-se a esse conceito a falta de voz, poder e independência dos pobres, que os sujeita à exploração; à propensão à doença; à falta de infraestrutura básica; à falta de ativos físicos, humanos, sociais e ambientais; e à maior vulnerabilidade e exposição ao risco (Crespo; Gurovitz, 2002).

Entretanto, no âmbito das favelas do Rio de Janeiro, podemos acrescentar, ao conceito formulado por Grespo e Gurovitz, que a multidimensionalidade da pobreza se intensifica, com a presença marcante da violência e da criminalidade com o tráfico de drogas, culminando frequentemente na transmissão intergeracional da pobreza.

No Brasil, até os anos 1980, a "cidadania" estava restrita aos trabalhadores inseridos no mercado formal de trabalho; era a "cidadania regulada"3 (Santos, 1994).

Conforme Draibe (2003), o reordenamento das políticas sociais no Brasil se constitui em dois ciclos reformistas nas décadas de 1980 e 1990. O primeiro ciclo se destacou pelo fortalecimento da ampliação dos direitos sociais instituídos na Constituição Federal de 1988, e o segundo ciclo, na década de 1990, caracterizou-se pelas reformas total e parcial que ocorreram no governo Fernando Henrique Cardoso.

As principais reformas dos programas sociais implantadas nos dois mandatos presidenciais de Fernando Henrique Cardoso (1995-1998 e 1999-2002) caracterizam-se por três programas: os universais, de saúde e educação; os de proteção ao trabalho e amparo no desemprego; e os programas de combate à pobreza.

Contudo, na década de 2000, o passivo social brasileiro ainda é expressivo; o combate à fome e à pobreza ganha centralidade na agenda política brasileira no governo de Luiz Inácio Lula da Silva (2003-2010). Entretanto, o Censo Demográfico realizado pelo IBGE em 2010 apontou a 
existência de mais de 16 milhões em situação de extrema pobreza no Brasil, metade no campo, outra parte nas cidades, especialmente nas grandes e metrópoles (Feres; Villatoro, 2005, s.p.).

Diante desta síntese histórica acerca das políticas sociais adotadas no Brasil entre as décadas de 1980 a 2010, de dimensão extrafamiliar Bird (2007), podemos sugerir que a "ausência" do Estado ou prioridades estabelecidas pelos governos, ao longo da história nessa arena deu a sua parcela de contribuição para a reprodução do ciclo da pobreza entre as gerações, inclusive nas favelas do Rio de Janeiro, desencadeando mecanismos produtores e transmissores da pobreza. Podemos destacar aspectos sociais e econômicos, limitação pessoal e aprisionamento local, que incluem desigualdades sistemáticas. Bem como outros elementos ressaltados no âmbito da pesquisa sobre transmissores da pobreza intergeracional, nomeadamente gravidez na adolescência, falta de cuidados na primeira infância, violência doméstica, desnutrição, falta de acesso a cuidados com a saúde, baixos níveis de educação, ativos domésticos e individuais de tomada de decisão, subsistência e estratégias de sobrevivência, normas culturais de gênero e práticas sociais de exploração de serviços, exposição e vulnerabilidade ao risco (Bird, 2007). Estes são alguns dos possíveis elementos geradores do ciclo intergeracional de pobreza que persistem nas famílias de moradoras e moradores dos territórios de favelas distantes de serem assistidos, conforme expresso desde a Declaração Universal dos Direitos Humanos de 1948, da qual o Brasil é signatário.

Portanto, este artigo tem por objetivo discutir uma abordagem intergeracional de pobreza por meio de uma metodologia qualitativa baseada no sentido atribuído e vivenciado das situações intra e extrafamiliar pelas pessoas moradoras do complexo de favelas de São João, no bairro do Engenho Novo, no Rio de Janeiro. A partir de narrativas captadas em entrevistas semiestruturadas sobre a geração antecessora e sobre a geração descendente, 29 pessoas opinaram sobre 23 variáveis que permitiram descrever a situação vivida e posteriormente aferir e interpretar a reprodução, ou não, da pobreza, tendo como padrão comparativo três gerações: a primeira geração (G1), formada por progenitores ou ascendentes; a segunda geração (G2) é a geração que foi entrevistada; e a terceira geração (G3) é de descendentes.

\section{COMPREENDENDO O CICLO INTERGERACIONAL DE POBREZA}

A transmissão intergeracional da pobreza é compreendida por Bird (2007) como uma consequência da ausência de transferências de investimentos necessários para educação, saúde e alimentação das pessoas, mas também pela ausência de políticas públicas que afetam o capital humano e social.

A contribuição de Bird (2007) remete para conhecimentos que versam sobre possíveis variáveis reprodutoras da pobreza entre gerações. Essas variáveis podem estar presentes tanto na dimensão intrafamiliar - renda, composição familiar - quanto na extrafamiliar - ausência de políticas públicas, fatores de ordem política, econômica e social, entre outros (Motta; Parente, 2018). 
Bird discute possíveis variáveis que afetam a pobreza na dimensão intrafamiliar, destaca a renda familiar, a composição do número de membros da família, a composição de ativos e patrimônio, educação de pais e mães, gravidez precoce e trabalho infantil, entre outros. Na dimensão extrafamiliar, as políticas públicas são o seu foco. Considera que a pobreza pode ocorrer em qualquer fase da vida; portanto, intervenções em fases mais vulneráveis devem ser consideradas, sobretudo no âmbito extrafamiliar, por meio de implantação de políticas públicas, conforme representado na Figura 1.

Figura 1. Mecanismos da reprodução da pobreza nas diversas fases do ciclo da vida Fonte: Autoras com base em Bird (2007) e Cain (2009).

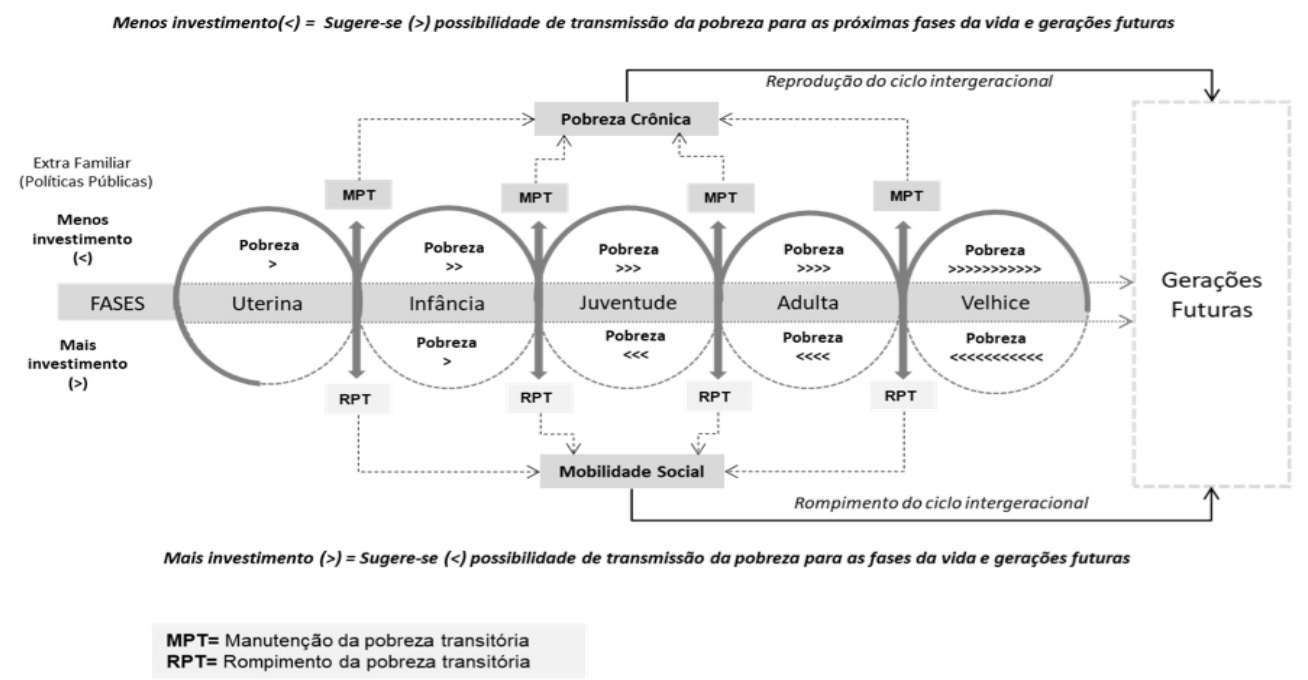

Conforme representado na Figura 1, investimentos em medidas sociais na dimensão extrafamiliar, bem como de políticas públicas nas primeiras fases do ciclo da vida da criança, inclusive na fase uterina, reduz as chances de a pobreza se perpetuar durante a juventude, adultez e velhice. Todavia, destacamos a importância do papel do Estado com a prestação de serviços essenciais e a implantação de políticas públicas robustas destinadas às pessoas empobrecidas em todas as fases do ciclo de vida. Programas de assistência à saúde, educação de qualidade, combate ao trabalho infantil, acesso ao mercado de trabalho, bem como políticas voltadas para velhice, são medidas de política pública que podem contribuir para o rompimento da pobreza transitória ${ }^{4}$, promover a mobilidade social ${ }^{5}$ e romper o ciclo intergeracional de pobreza. Ao contrário, a ausência do Estado prestando serviços essenciais em quaisquer fases do ciclo de vida aumenta a possibilidade de manutenção da pobreza transitória e condução para o nível de pobreza crônica ${ }^{6}$ contribui, portanto, para a reprodução do ciclo da pobreza para as futuras gerações, de acordo com Hulme e Shepherd (2003).

\section{ESTRATÉGIA METODOLÓGICA QUALITATIVA}

Este artigo resulta de um projeto de tese aprovado pelos comitês de ética da Universidade do Estado do Rio de Janeiro e da Secretaria Municipal de Saúde do Rio de Janeiro, além de submetido 
à Plataforma Brasil e aprovado em 29 de junho de 2016, sob o CAAE 58239616.4.0000.5282. Os estudos sobre a transmissão intergeracional da pobreza sugerem a adequação da pesquisa qualitativa (Bird, 2010). Essa foi a abordagem adotada num procedimento metodológico de cariz exploratório que utilizou a entrevista semiestruturada com 29 pessoas do complexo do São João, que é constituído por um total de 1.874 domicílios. O objetivo foi descrever o sentimento de pobreza comparado entre gerações, de modo a aferir a tendência (ou não) de reprodução a que estão submetidos os moradores e as moradoras das favelas do Rio de Janeiro numa época de generalização e intensificação das políticas sociais de combate à pobreza no Brasil.

\subsection{Recolha de dados}

O estudo abrangeu três gerações: uma de forma direta, por meio das narrativas construídas nas entrevistas semiestruturadas realizadas com pessoas cujo requisito era dispor de condições psíquicas e memória para responder às perguntas sobre duas gerações familiares com precisão: a dos seus progenitores e a dos seus descendentes; as duas gerações a montante e a jusante foram captadas indiretamente por meio das representações veiculadas nos discursos da geração entrevistada. Antecipando-se às possíveis dificuldades de acesso da pesquisadora por eventuais momentos de conflitos armados inerentes ao contexto da favela, optou-se por realizar as entrevistas na Clínica da Família Cabo Edney Canazaro de Oliveira, sob a coordenadoria da Secretaria Municipal de Saúde do Rio de Janeiro, sediada nas imediações da favela; o atendimento destina-se prioritariamente a moradoras e moradores do complexo do São João. A grande frequência de moradoras e moradores para atendimento na clínica contribuiu para atingir o número suficiente de respondentes provenientes de diversas partes do complexo de favelas. Entre as principais implicações resultantes do contexto destacam-se as interrupções da entrevista para a realização dos procedimentos agendados e a inicial vinculação, pelas e pelos respondentes, da relação da pesquisa com os serviços oferecidos pela clínica, podendo a entrevista influenciar no futuro atendimento, bem como a maior frequência de mulheres nesse contexto de assistência, o que se traduziu numa menor participação de homens. As pessoas eram convidadas a participar da pesquisa aleatoriamente. Ao ser convidada/o, a/o respondente recebeu inicialmente as informações sobre o objetivo do estudo. Em seguida, para identificar se a pessoa tinha o perfil necessário para participar da entrevista, realizou-se uma triagem básica formulando três perguntas: confirmação do local de moradia, idade das/os filhas/os e disponibilidade de tempo para responder às perguntas. Foram desconsideradas, para a realização das entrevistas, pessoas que moravam no entorno do complexo do São João que não correspondiam aos setores censitários do complexo de favelas do São João; pessoas que não tinham filhos com idade superior a 10 anos de idade; pessoas que não tinham disponibilidade de tempo para a realização da entrevista ou que não se interessaram pelo estudo.

Finalmente, e seguindo as diretrizes expressas na Resolução $n^{\circ} 466 / 2012$, foi explicado em linguagem corrente o Termo de Consentimento Livre e Esclarecido (TCLE). A moradora ou o morador assinou o TCLE e recebeu uma cópia do documento, autorizando também a gravação das entrevistas. Durante os 30 dias de entrevistas, aproximadamente 50 pessoas foram abordadas 
para participar da pesquisa. Dentre elas, 32 se encaixaram no perfil e participaram da entrevista. Três entrevistas foram eliminadas por não ter sido possível encontrar um raciocínio claro e lógico, tendo-se chegado ao final do registro com informações insuficientes e incoerentes para o tratamento dos dados.

\subsection{Análise dos dados}

Como afirma Jean-Pierre Deslauriers, citado em Guerra (2006), a expressão "métodos qualitativos"

não tem um sentido preciso em ciências sociais. No melhor dos casos, designa uma variedade de técnicas interpretativas que têm por fim descrever, decodificar, traduzir certos fenômenos sociais que se produzem mais ou menos naturalmente. Essas técnicas dão mais atenção ao significado desses fenômenos do que à sua frequência (1997, p. 294).

Foi nessa perspectiva que este estudo adotou a técnica interpretativa, baseada numa proposta de raciocínio sistemático que era feita aos entrevistados para comparar as três gerações a propósito de 23 variáveis (Quadro 1), de modo aferir possíveis reprodutores, ou não, da pobreza nas dimensões intrafamiliar e extrafamiliar, o que permitiu uma melhor compreensão do fenômeno investigado. O Quadro 1 resulta da definição das escalas de medição das 23 variáveis e tem como objetivo identificar a tendência para a melhoria (ascensão), para a manutenção (estacionária) ou para degradação (descendência) em cada uma das variáveis.

Quadro 1. Escalas de medição das variáveis.

\begin{tabular}{|c|c|c|}
\hline \multicolumn{3}{|c|}{ Variáveis x Escalas } \\
\hline \multicolumn{3}{|c|}{ Variável - Dimensão intrafamiliar } \\
\hline MENOS - IGUAL - MAIS & PIOR - IGUAL - MELHOR & MENOR - IGUAL - MAIOR \\
\hline $\begin{array}{l}\text { 01: "Anos de estudos"; 05: "Pat- } \\
\text { rimônio"; 07: "Gravidez na ado- } \\
\text { lescência"; 09: "Deficiência física ou } \\
\text { mental"; 10: "Violência intrafamiliar } \\
\text { e/ou extrafamiliar"; } 12: \text { "Membros } \\
\text { da família que trabalha(ra)m formal- } \\
\text { mente"; 13: "Membros da família que } \\
\text { trabalha(ra)m informalmente"; 14: } \\
\text { "Membros da família sem fonte de } \\
\text { renda" e 15: "Casos de privações". }\end{array}$ & $\begin{array}{l}\text { 02: "Tipo de Moradia"; } \\
\text { 04: "Alimentação"; 08: } \\
\text { "Saúde" e 11: "Condições } \\
\text { de Trabalho". }\end{array}$ & $\begin{array}{l}\text { 03: "Renda Familiar" e 06: } \\
\text { "Número de filhos". }\end{array}$ \\
\hline \multicolumn{3}{|c|}{ Variável - Dimensão extrafamiliar } \\
\hline \multicolumn{3}{|c|}{ MENOS - IGUAL - MAIS } \\
\hline $\begin{array}{l}\text { 16: "Acesso a programas de governo" } \\
\text { à conta bancária"; } 20 \text { : "Acesso a água } \\
\text { "Acesso ao mercado de trabalho form }\end{array}$ & $\begin{array}{l}\text { 17: "Acesso à educação"; } 1 \\
\text { luz e saneamento básico"; } \\
\text { al" e 23: "Acesso a lazer". }\end{array}$ & $\begin{array}{l}\text { "Acesso à saúde"; 19: "Acesso } \\
\text { "Acesso à moradia"; 22: }\end{array}$ \\
\hline
\end{tabular}

Fonte: Elaborado pelas autoras. 
Além da escala de análise, para cada uma das 23 variáveis foram definidos o seu objetivo para o estudo e o critério a ser adotado, conforme exemplificado no Quadro 2.

Quadro 2. Critério de análise: Variável 01.

\begin{tabular}{|l|l|}
\hline \multicolumn{2}{|c|}{ Variável 01: “Anos de estudos” } \\
\hline $\begin{array}{l}\text { Definição: Indicar o tipo de mobilidade entre as gerações G2 x G1 e } \\
\text { G3 x G2 em relação aos anos de estudo. }\end{array}$ & $\begin{array}{l}\text { Escala de análise: } \\
\text { MENOS - IGUAL - MAIS }\end{array}$ \\
\hline $\begin{array}{l}\text { Critério de análise G1: Foram considerados na análise desta variável os anos de estudos do } \\
\text { pai, da mãe ou do responsável com mais anos de estudos. }\end{array}$ \\
\hline $\begin{array}{l}\text { Critério de análise G2 e G3: Foram considerados na análise desta variável os anos de estudo } \\
\text { de todo(s) o(s) membro(s) da G2 e G3. }\end{array}$ \\
\hline $\begin{array}{l}\text { Não foram considerados na análise desta variável os membros da G3 falecidos e quando o re- } \\
\text { spondente G2 não soube informar. }\end{array}$ \\
\hline
\end{tabular}

Fonte: Elaborado pelas autoras.

Com base nesses primeiros critérios de análise e nas respostas informadas pela/o entrevistada/o, foi aplicada a matriz tipológica I de mobilidade intergeracional, em que se avalia a tendência para a melhoria (ascensão), para a manutenção (estacionário) ou para degradação (descendência) (Quadro 3).

Quadro 3. Matriz I - Tipos de mobilidade intergeracional

\begin{tabular}{|c|c|c|c|}
\hline \multicolumn{4}{|c|}{ Tipos de mobilidade intergeracional (variável x membros da família) } \\
\hline Gerações & Ascendência & \begin{tabular}{|c|} 
Estacionária \\
\end{tabular} & Descendência \\
\hline \multirow{2}{*}{$\begin{array}{l}\text { G2 } \\
\mathbf{X} \\
\mathbf{G 1}\end{array}$} & \multirow{2}{*}{$\begin{array}{l}\text { A = Ascendente: } \\
\text { indica a ascensão } \\
\text { da variável anali- } \\
\text { sada em G2 em } \\
\text { relação a G1. }\end{array}$} & $\begin{array}{l}\text { EP = Estacionária positiva: indica } \\
\text { a ausência de mobilidade positiva da } \\
\text { variável analisada em G2 em relação } \\
\text { a G1. }\end{array}$ & \multirow{2}{*}{$\begin{array}{l}\text { D = Descendente: indica } \\
\text { descensão da variável } \\
\text { analisada em G2 em rela- } \\
\text { ção a G1. }\end{array}$} \\
\hline & & $\begin{array}{l}\text { EN = Estacionária negativa: indica } \\
\text { ausência de mobilidade negativa na } \\
\text { variável analisada em G2 em relação } \\
\text { a G1. }\end{array}$ & \\
\hline
\end{tabular}

Fonte: Elaborado pelas autoras. 
Quadro 3. Matriz I - Tipos de mobilidade intergeracional

\begin{tabular}{|c|c|c|c|}
\hline \multirow{5}{*}{$\begin{array}{c}\text { G3 X G2 } \\
\text { Níveis }\end{array}$} & $\begin{array}{l}\text { AE = Ascensão } \\
\text { elevada: indica as- } \\
\text { censão da variável } \\
\text { analisada em } \\
100 \% \text { dos mem- } \\
\text { bros da G3 em } \\
\text { relação a G2. }\end{array}$ & \multirow{3}{*}{$\begin{array}{l}\text { EE = Estacionária elevada: indica } \\
\text { a ausência de mobilidade positiva ou } \\
\text { negativa da variável analisada em } \\
100 \% \text { dos membros da G3 em relação } \\
\text { a G2. }\end{array}$} & $\begin{array}{l}\text { DE = Descensão el- } \\
\text { evada: indica descensão } \\
\text { da variável analisada em } \\
100 \% \text { dos membros da } \\
\text { G3 em relação a G2. }\end{array}$ \\
\hline & $\begin{array}{l}\text { AM = Ascensão } \\
\text { moderada: indica } \\
\text { a ascensão da va- } \\
\text { riável analisada = } \\
\text { ou > em } 50 \% \text { dos } \\
\text { membros da G3 } \\
\text { em relação a G2. }\end{array}$ & & $\begin{array}{l}\text { DM = Descensão mode- } \\
\text { rada: indica descensão } \\
\text { da variável analisada < } \\
\text { em } 50 \% \text { dos membros } \\
\text { da G3 e estacionária em } \\
\text { parte dos membros da } \\
\text { G3 e sem ascensão em } \\
\text { nenhum membro da G3 } \\
\text { em relação a G2. }\end{array}$ \\
\hline & $\begin{array}{l}\text { AB = Ascensão } \\
\text { baixa: indica a as- } \\
\text { censão da variável } \\
\text { analisada < em } \\
50 \% \text { dos membros } \\
\text { da G3 em relação } \\
\text { a G2. }\end{array}$ & & $\begin{array}{l}\text { DB = Descensão bai- } \\
\text { xa: indica descensão da } \\
\text { variável analisada < em } \\
50 \% \text { dos membros da G3 } \\
\text { e estacionária em parte } \\
\text { dos membros da G3, sem } \\
\text { ascensão em nenhum } \\
\text { membro da G3 em rela- } \\
\text { ção a G2. }\end{array}$ \\
\hline & \multicolumn{3}{|c|}{ Escolar } \\
\hline & \multicolumn{3}{|c|}{$\begin{array}{l}\text { DT = Descensão transitória: indica a descensão da variável analisada, anos de estu- } \\
\text { dos, e parte de membros da G3 encontram-se regularmente matriculados e frequentes, } \\
\text { com possibilidade de ascensão nos anos de estudo em relação a G2. }\end{array}$} \\
\hline
\end{tabular}

Fonte: Elaborado pelas autoras.

Na etapa de aplicação na Matriz I, extraímos o tipo de mobilidade entre as gerações G2 x G1 e o tipo de mobilidade entre as gerações $\mathrm{G} 3 \times \mathrm{G} 2$. Com base nas respostas do/a entrevistado/a, fizemos a análise comparativa da mobilidade da variável entre as gerações $\mathrm{G} 2$ x G1 e o tipo de mobilidade entre as gerações G3 x G2. Assim, obtivemos o tipo de mobilidade intergeracional da respectiva variável para as respectivas famílias. Em seguida aglutinamos os dois tipos de mobilidade: a extraída nas gerações G2 x G1 e a extraída nas gerações G3 x G2; obtivemos o tipo de mobilidade intergeracional da variável analisada entre as três gerações $(\mathrm{G} 1 \times \mathrm{G} 2 \times$ G3), conforme exemplo do Quadro 4, Análise da mobilidade geracional da variável 01: "Anos de estudos" das três gerações da Família 01. 


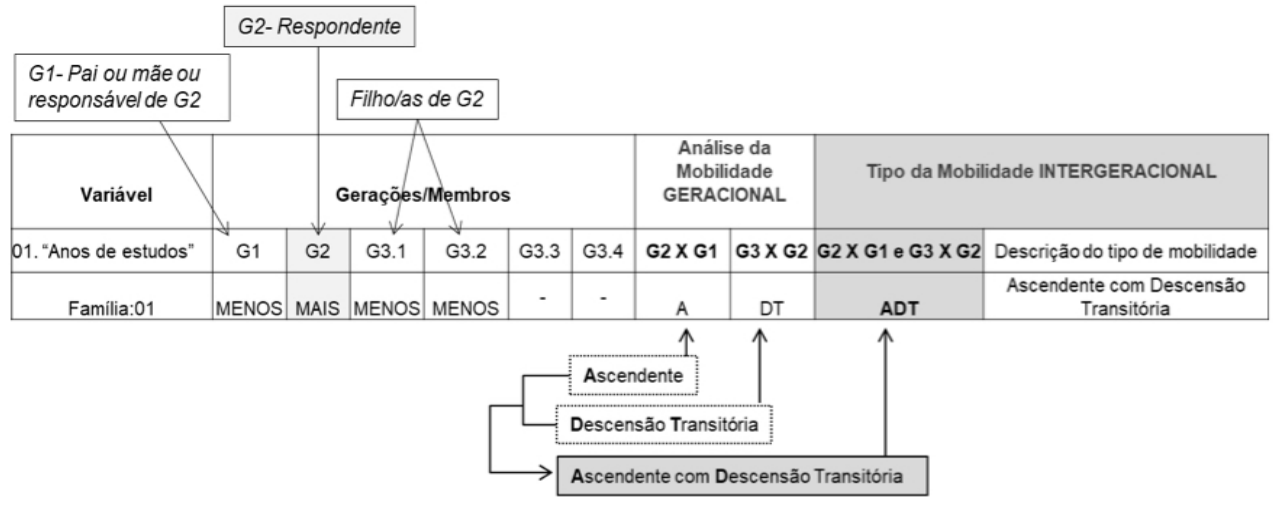

Quadro 4. Tipo de modalidade geracional da variável 01: "Anos de estudos" - Família 01. Fonte: Elaborado pelas autoras.

Com base na aglutinação dos dois tipos de mobilidades, partimos para a classificação da mobilidade intergeracional, sendo possível estabelecer três classificações sistematizadas no Quadro 5: i) classificação "positiva", que representa tipos de mobilidade de variáveis com ascensão, descensão e estacionaridade, de nível elevado e descensão transitória, na análise das gerações G3 x G2 com características positivas. Ex.: ascensão elevada em anos de estudos; ii) classificação "estacionária", que representa tipos de mobilidade de variáveis com ascensão, descensão e estacionaridade, de nível moderado e baixo e descensão transitória, na análise das gerações G3 x G2, com características positivas e negativas. Ex.: ascensão baixa em anos de estudos ou descensão baixa de gravidez precoce; iii) classificação "negativa", que representa tipos de mobilidade de variáveis com ascensão, descensão e estacionárias de nível elevado, na análise das gerações G3 x G2, com características negativas. Ex.: ascensão elevada da gravidez na adolescência.

Quadro 5. Matriz II - Classificação da mobilidade intergeracional da variável

\begin{tabular}{|l|l|l|}
\hline \multicolumn{3}{|c|}{ Classificação do tipo de mobilidade da variável x gerações/membros } \\
\hline \multicolumn{1}{|c|}{ Positiva } & \multicolumn{1}{|c|}{ Estacionária } & \multicolumn{1}{c|}{ Negativa } \\
\hline $\begin{array}{l}\text { MP = Mobilidade positiva: } \\
\text { indica ascensão, descensão } \\
\text { e estacionaridade da variável } \\
\text { analisada com características } \\
\text { positivas. }\end{array}$ & $\begin{array}{l}\text { ME = Mobilidade esta- } \\
\text { cionária: indica ascensão, } \\
\text { descensão e estacionaridade } \\
\text { da variável analisada com } \\
\text { características positivas e/ou } \\
\text { negativas. }\end{array}$ & $\begin{array}{l}\text { MN = Mobilidade negativa: } \\
\text { indica ascensão, descensão } \\
\text { e estacionaridade da variável } \\
\text { analisada com características } \\
\text { negativas. }\end{array}$ \\
\hline \multicolumn{3}{|c|}{ Critério de análise } \\
\hline
\end{tabular}

Fonte: Elaborado pelas autoras. 


\section{Quadro 5. Matriz II - Classificação da mobilidade intergeracional da variável}

\begin{tabular}{|c|c|c|}
\hline $\begin{array}{l}\text { Para a composição do tipo } \\
\text { de mobilidade intergeracional } \\
\text { POSITIVA foram considera- } \\
\text { dos os tipos de mobilidades } \\
\text { intergeracional de variáveis } \\
\text { com Ascensão, Descensão e } \\
\text { Estacionária com mobilidade } \\
\text { intergeracional POSITIVA, de } \\
\text { nível elevado, e Descensão } \\
\text { Transitória, dos membros da } \\
\text { G3 em relação a G2. }\end{array}$ & $\begin{array}{l}\text { Para a composição do tipo } \\
\text { de mobilidade intergeracional } \\
\text { ESTACIONÁRIA foram consid- } \\
\text { erados os tipos de mobilidade } \\
\text { intergeracional de variáveis } \\
\text { com Ascensão, Descensão } \\
\text { com mobilidade intergeracional } \\
\text { POSITIVA ou NEGATIVA, de } \\
\text { nível moderado e baixo dos } \\
\text { membros da G3 em relação a } \\
\text { G2. }\end{array}$ & $\begin{array}{l}\text { Para a composição do tipo } \\
\text { de mobilidade intergeracional } \\
\text { da classe NEGATIVA foram } \\
\text { considerados os tipos de } \\
\text { mobilidade intergeracional de } \\
\text { variáveis com Ascensão, De- } \\
\text { scensão e Estacionária com } \\
\text { mobilidade intergeracional } \\
\text { NEGATIVA, de nível elevado } \\
\text { dos membros da G3 em rela- } \\
\text { ção a G2. }\end{array}$ \\
\hline
\end{tabular}

Fonte: Elaborado pelas autoras.

A título de exemplo, o Quadro 6 traz as etapas percorridas até a classificação do tipo de mobilidade intergeracional da variável 01: "Anos de estudos", da Família 01.

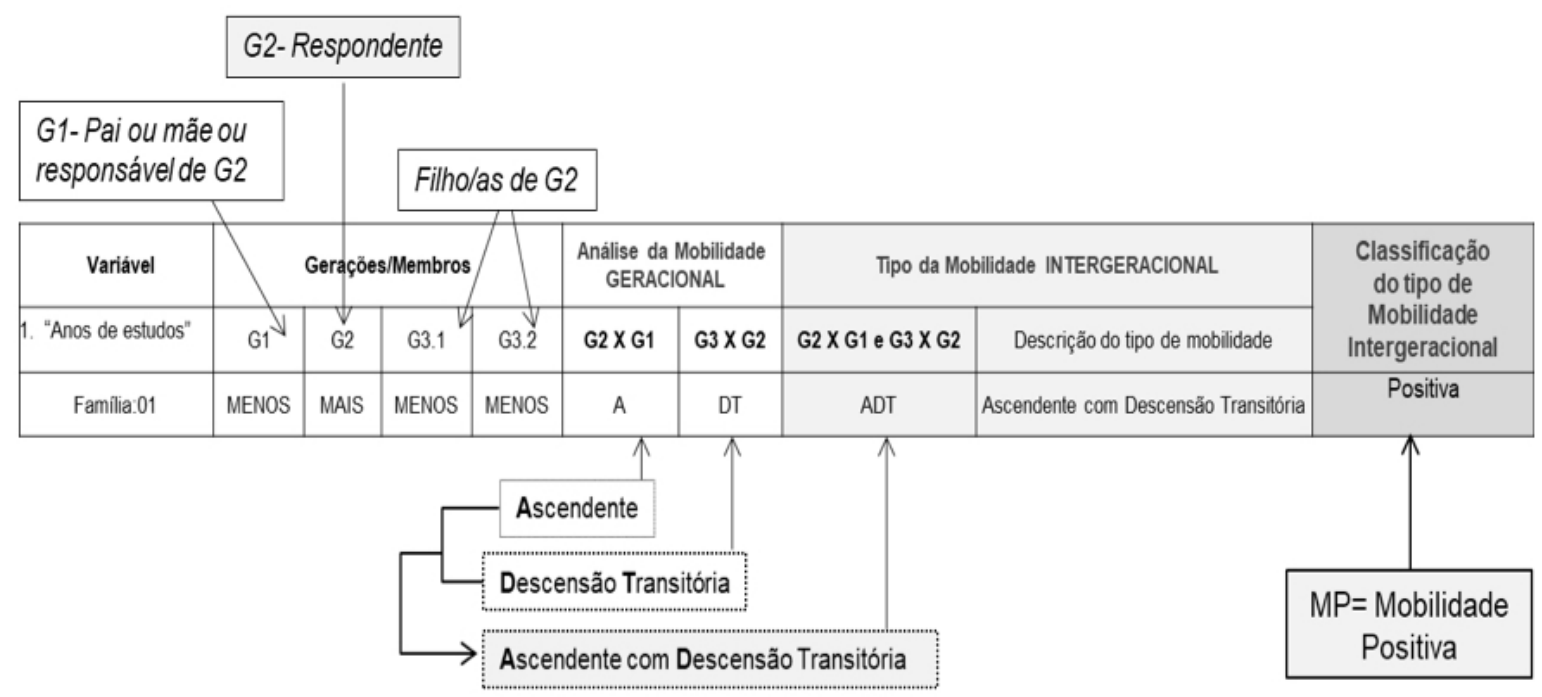

Quadro 6. Classificação do tipo de mobilidade intergeracional da Variável 01: "Anos de estudos” - Família 01

Fonte: Elaborado pelas autoras. 
Por fim, com o objetivo de obter a classificação do tipo de mobilidade intergeracional de cada família do estudo, foi criada a terceira matriz de análise, constituída por três tipos de mobilidade intergeracional familiar: a "ascendente baixa", a "ascendente moderada" e a "ascendente elevada", conforme apresentado a seguir.

Quadro 7. Matriz III - Classificação do tipo de modalidade intergeracional familiar

\begin{tabular}{|c|c|c|}
\hline \multicolumn{3}{|c|}{ Classificação da mobilidade intergeracional familiar } \\
\hline Ascendente baixa & Ascendente moderada & Ascendente elevada \\
\hline $\begin{array}{l}\text { MAB = Mobilidade ascen- } \\
\text { dente baixa: indica ascen- } \\
\text { são com mobilidade posi- } \\
\text { tiva, abaixo de } 50 \% \text { do total } \\
\text { variáveis analisadas, em G3 } \\
\text { em relação a G2. }\end{array}$ & $\begin{array}{l}\text { MAM = Mobilidade ascendente } \\
\text { moderada: indica ascensão } \\
\text { com mobilidade positiva ou } \\
\text { negativa entre } 50 \% \text { até } 70 \% \text { do } \\
\text { total de variáveis analisadas em } \\
\text { G3 em relação a G2. }\end{array}$ & $\begin{array}{l}\text { MAE = Mobilidade ascendente } \\
\text { elevada: indica ascensão com mo- } \\
\text { bilidade positiva, acima de } 70 \% \text { do } \\
\text { total de variáveis analisadas, em } \\
\text { G3 em relação a G2. }\end{array}$ \\
\hline \multicolumn{3}{|c|}{ Critério de análise } \\
\hline $\begin{array}{l}\text { Para a composição da } \\
\text { categoria de mobilidade } \\
\text { intergeracional ASCEN- } \\
\text { DENTE BAIXA foram con- } \\
\text { sideradas as classificações } \\
\text { de mobilidade intergeracio- } \\
\text { nal das variáveis com As- } \\
\text { censão, Descensão e Es- } \\
\text { tacionária com mobilidade } \\
\text { NEGATIVA de nível elevado } \\
\text { dos membros da G3 em } \\
\text { relação a G2. }\end{array}$ & $\begin{array}{l}\text { Para a composição da categoria } \\
\text { de mobilidade intergeracional } \\
\text { ASCENDENTE MODERADA } \\
\text { foram consideradas as classifi- } \\
\text { cações de mobilidade intergera- } \\
\text { cional das variáveis com Ascen- } \\
\text { são, Descensão com mobilidade } \\
\text { POSITIVA ou NEGATIVA de nív- } \\
\text { el moderado e baixo e Descen- } \\
\text { são Transitória, dos membros da } \\
\text { G3 em relação a G2. }\end{array}$ & $\begin{array}{l}\text { Para a composição da categoria de } \\
\text { mobilidade intergeracional ASCEN- } \\
\text { DENTE ELEVADA foram considera- } \\
\text { das as classificações de mobilidade } \\
\text { intergeracional das variáveis com } \\
\text { Ascensão, Descensão e Esta- } \\
\text { cionária com mobilidade POSITIVA } \\
\text { de nível elevado dos membros da } \\
\text { G3 em relação a G2. }\end{array}$ \\
\hline
\end{tabular}

Fonte: Elaborado pelas autoras

Após a aplicação da Matriz III, extraímos o percentual correspondente e classificamos o tipo de mobilidade intergeracional da família analisada. Para identificar a classificação da mobilidade intergeracional de cada família, identificamos o percentual do total de variáveis com classificação de mobilidade Intergeracional "positiva", conforme exemplo da análise da Família 01. 
Quadro 8. Cálculo percentual para as classificações de mobilidade intergeracional da Família 01.

\begin{tabular}{|l|c|c|}
\hline \multicolumn{1}{|c|}{$\begin{array}{c}\text { Cálculo percentual do tipo de mobilidade in- } \\
\text { tergeracional - Família: 01 }\end{array}$} & $\begin{array}{c}\text { Dimensão intra- } \\
\text { familiar }\end{array}$ & Dimensão extrafamiliar \\
\hline Total de variáveis do estudo & 15 & 8 \\
\hline Total de variáveis "positiva" identificadas na família & 9 & 4 \\
\hline *Total de variáveis não analisadas & 6 & 4 \\
\hline Total de variáveis analisadas & 9 & 4 \\
\hline$\%$ de mobilidade intergeracional positiva & $100 \%$ & $100 \%$ \\
\hline \multicolumn{2}{|c|}{ Ascendente elevada } \\
\hline $\begin{array}{l}\text { *As variáveis em que não foi possível fazer a análise comparativa entre as gerações por falta de dados informados } \\
\text { pelo/a respondente (G2) não foram consideradas para o cálculo do percentual. }\end{array}$ \\
\hline
\end{tabular}

Fonte: Elaborado pelas autoras.

O percentual foi extraído com base no total de variáveis analisadas. As variáveis em que não foi possível fazer a análise comparativa G3 x G2 por falta de dados não informados pelo/a respondente (G2) não foram consideradas para o cálculo do percentual (no quadro estão assinalados como "não analisado"). Após análise, para classificação do tipo de mobilidade intergeracional de todas as variáveis, calculamos o percentual do total das variáveis com classificação de mobilidade Intergeracional "positiva". Com base no cálculo do percentual do total das variáveis identificadas na análise comparativa de $\mathrm{G} 3 \times \mathrm{G} 2$, aplicamos o critério de análise da terceira matriz de classificação do tipo de mobilidade intergeracional familiar (Quadro 7).

\section{RESULTADOS DO ESTUDO}

Após as etapas de análises, obtivemos a classificação do tipo de mobilidade intergeracional familiar das 29 famílias entrevistadas. Para apresentar o resultado final, optamos pela análise tipológica dos dados, que consiste na formação de seis tipos de famílias agrupadas por semelhanças por apresentarem as mesmas classificações do tipo de mobilidade intergeracional familiar nas dimensões intrafamiliar e extrafamiliar.

Conforme afirmam Barros e Carvalho (2006) sobre os desafios acerca da elaboração de indicadores para mensuração a dimensão da pobreza na perspectiva na análise multidimensional,

ainda estamos longe de saber normativamente quais são as dimensões mais relevantes da pobreza e também distantes de ter definido os melhores indicadores para representar cada dimensão. Em geral, a definição das dimensões e dos indicadores segue uma estratégia eminentemente pragmática e empírica em que pesam a disponibilidade e a confiabilidade das informações. (...) 
Dada a complexidade de se trabalhar com um número tão elevado de indicadores e a necessidade de ordenar a pobreza das famílias, comunidades, municípios ou estados, surge a necessidade de se criar indicadores sintéticos. Estes indicadores buscam sintetizar, em um único número, a informação de diversos indicadores básicos (Barros; Carvalho; Franco, 2006).

O resultado da análise tipológica consiste na apresentação do resultado dos dados apresentando os índices de classificação das famílias conforme o tipo de mobilidade intergeracional nas dimensões intrafamiliar e intrafamiliar (Quadro 9).

Quadro 9 - Índices de mobilidade familiar

\begin{tabular}{|l|c|c|}
\hline \multicolumn{1}{|c|}{ Dimensão intrafamiliar } & Total & $\%$ \\
\hline Mobilidade ascendente elevada & 4 & $14 \%$ \\
\hline Mobilidade ascendente moderada & 12 & $41 \%$ \\
\hline Mobilidade ascendente baixa & 13 & $45 \%$ \\
\hline \multicolumn{1}{|c|}{ Dimensão intrafamiliar } & Total & $\%$ \\
\hline Mobilidade ascendente elevada & 10 & $34 \%$ \\
\hline Mobilidade ascendente moderada & 18 & $62 \%$ \\
\hline Mobilidade ascendente baixa & 1 & $3 \%$ \\
\hline Total & 29 & $100 \%$ \\
\hline
\end{tabular}

Fonte: Elaborado pelas autoras.

A leitura do Quadro 9 mostra que, na dimensão intrafamiliar, quatro famílias foram classificadas com o tipo de mobilidade "ascendente elevada", o que significa que essas famílias tiveram mobilidade com ascensão positiva acima de $70 \%$ do total de variáveis; doze famílias foram classificadas com o tipo de mobilidade ascendente moderada, ou seja, tiveram mobilidade com ascensão positiva ou negativa acima de $50 \%$ e abaixo de $70 \%$ do total de variáveis; treze famílias foram classificadas com o tipo de mobilidade ascendente baixa, ou seja, apresentaram mobilidade com ascensão positiva abaixo de $50 \%$ do total de variáveis.

Na dimensão extrafamiliar, temos que dez famílias foram classificadas com mobilidade do tipo ascendente elevada, ou seja, tiveram mobilidade com ascensão positiva acima de $70 \%$ do total de variáveis; dezoito famílias foram classificadas com mobilidade ascendente moderada, o que significa que tiveram mobilidade com ascensão positiva ou negativa acima de $50 \%$ e abaixo de $70 \%$ do total de variáveis; uma família foi classificada com o tipo de mobilidade ascendente baixa, isto é, apresentou mobilidade com ascensão positiva abaixo de 50\% do total de variáveis.

Comparando as dimensões intra e extrafamiliar, verificamos que a mobilidade intergeracional familiar é maior na dimensão extrafamiliar, pois o total de famílias classificadas com o tipo de mobilidade ascendente moderada e ascendente elevada são dezoito e dez famílias, respectivamente; na dimensão intrafamiliar o total de famílias classificadas com mobilidade do tipo ascendente elevada e moderada se reduziu para quatro e doze famílias, respectivamente, o que demonstra variações entre o acesso às políticas públicas e seus efeitos na vida das famílias. 


\section{CONCLUSÕES E CONSIDERAÇÕES FINAIS}

A nossa proposta de avaliação segue a perspectiva construtivista que se afirmou nos anos de 1970 na avaliação de programas sociais, em conflito com a perspectiva positivista-experimental anterior, que propõe a análise qualitativa e o envolvimento dos atores com as políticas públicas nessa avaliação (Serapioni, 2016). No Brasil, as avaliações de impacto dos programas sociais são em geral realizadas utilizando os macro e microdados da Pesquisa Nacional por Amostra de Domicílios (PNAD); todavia, os modelos adotados privilegiam dados e números cuja configuração não permite a aproximação da realidade social, conforme afirma Minayo (2007) quando expressa que a abordagem qualitativa possibilita a compreensão da realidade social, a qual não pode ser simplesmente interpretada estatisticamente, pois a interpretação baseada apenas em números não permite a aproximação do real para a compreensão da diversidade e da heterogeneidade do mundo real. Este estudo exploratório possibilitou, por meio de rigoroso e sistemático critério de análise qualitativa do conteúdo das entrevistas, a compreensão da pobreza entre as gerações G1, G2 e G3, tomando por base as avaliações dos envolvidos.

Foi na perspectiva do rompimento da situação e do processo da pobreza no paradigma multidimensional que este estudo adotou o conceito multidimensional de pobreza (Crespo; Gurovitz, 2002) para refletir sobre as representações das condições de vida a que estão submetidos os moradores e as moradoras das favelas do Rio de Janeiro, bem como para aferir como são sentidas as políticas sociais de combate à pobreza no Brasil.

Concluímos que os fatores promotores do ciclo intergeracional da pobreza nesses territórios estão presentes tanto na dimensão intrafamiliar quanto na dimensão extrafamiliar, conforme remete Bird (2007), apesar de haver tendência de mobilidade ascendente mais visível na dimensão extrafamiliar do que na dimensão familiar. Esse fato resulta de alguma forma das políticas sociais públicas generalistas. Portanto, parece-nos que, para universalização e efetividade das políticas públicas destinadas ao rompimento da pobreza nesses territórios, são necessários estudos aprofundados sobre as dinâmicas sociais específicas inerentes ao contexto das favelas cariocas para subsidiar a formulação de medidas na dimensão intrafamiliar consubstanciadas nas particularidades do local. Os dados sugerem que as políticas públicas universais destinadas a esse fim contribuem para a produção de efeitos positivos na vida das pessoas e tiram as pessoas da condição de extrema pobreza, mas não contribuem para o rompimento da pobreza em suas múltiplas dimensões e para a mobilidade social, podendo perpetuar-se na pobreza ao longo dos ciclos de vida da pessoa e ser transmitida para as gerações futuras.

Na dimensão intrafamiliar, elementos como a composição e o número de membros da família, a baixa composição da renda familiar, a gravidez na adolescência, a negligência na formação dos filhos praticada por pai ou mãe ou responsável, a violência dentro e fora do ambiente familiar, o baixo nível de escolaridade, entre outros, contribuíram de forma negativa na vida das pessoas do estudo. Na dimensão extrafamiliar, as dinâmicas sociais vivenciadas por seus moradores e moradoras com características próprias desses territórios e menos presentes em outros contextos de pobreza, como a pobreza em outros grandes centros urbanos em que os conflitos armados, o 
tráfico de drogas, a presença de milícias não são tão marcantes como nas favelas cariocas, além da discriminação social e dificuldades para inserção produtiva formal ou legal. Somos conduzidas a afirmar que as condições de vida e a exclusão social e produtiva a que estão submetidos os moradores e as moradoras das favelas do Rio de Janeiro levam à acumulação de obstáculos e dificuldades que contribuem para a reprodução do ciclo intergeracional de pobreza.

Os estudos científicos em profundidade, alicerçados em metodologias de investigação na e para a ação (Esteves, 1986), e a redução do distanciamento entre o mundo acadêmico e os formuladores de políticas públicas são avanços necessários sobretudo para promover um conhecimento mais autêntico, próximo e coproduzido nesses territórios e aplicar orientações metodológicas que sejam capazes não só de mensurar a pobreza em suas múltiplas dimensões e intensidades como também de compreender as dinâmicas sociais inerentes ao contexto das favelas para subsidiar a formulação de políticas públicas cuja configuração leve em consideração a realidade do local.

A partir dos resultados apontados, podemos inferir que, no âmbito das favelas do Rio de Janeiro, a educação é um dos pontos críticos e mais relevantes quando analisada na perspectiva da sua relação como determinante positiva para a inserção no mercado de trabalho, pois muitos não conseguem concluir seus estudos, conforme evidenciou esta pesquisa; o baixo nível de escolaridade atinge as três gerações.

A análise dos dados nos revela variações entre o acesso às políticas públicas e seus efeitos na vida das famílias e que a principal semelhança entre a dinâmica social e os grupos de famílias do estudo concentrou-se no baixo nível de escolaridade dos membros paternos da G1 e na reprodução do baixo nível de escolaridade nas três gerações (G1, G2 e G3). Embora membros da geração $\mathrm{G} 3$ tenham tido mais acesso à educação, bem como a programas sociais, os resultados sugerem que as dinâmicas sociais vivenciadas no contexto dessas famílias, como a negligência materna e paterna, gravidez na adolescência e a violência, contribuem para a produção de elementos negativos; logo, para a reprodução do ciclo intergeracional da pobreza.

A pobreza, conforme destaca Bird (2007), pode ser reproduzida em qualquer fase do ciclo de vida, mas a intervenção com medidas sociais nos primeiros anos de uma criança, mesmo ainda na fase uterina, pode reduzir as chances de uma criança ser pobre ao longo da vida; portanto, vale destacar a importância de formulação de políticas públicas que atuem preventivamente nessa fase e em outras fases do ciclo de vida das pessoas, inclusive na velhice. Atuar de forma preventiva significa contribuir para a melhoria de condições de vidas das pessoas, para o rompimento da pobreza transitória, crônica, para o rompimento do ciclo intergeracional da pobreza e, sobretudo, para a redução da dependência das pessoas dos programas de transferências de renda ao longo da vida.

Discorremos sobre recomendações de possíveis medidas para ultrapassar as limitações que impedem o rompimento do ciclo intergeracional da pobreza nas favelas cariocas. Sugerimos como primeiro passo a retomada do Estado, no âmbito da segurança pública, a reformulação de um novo modelo de unidade de polícia pacificadora ${ }^{7}$, cuja configuração contemple a preparação do 
efetivo e, sobretudo, proporcionar melhores condições de trabalho para a atuação da segurança pública no território.

A segunda proposição discorre sobre a implantação de políticas públicas de combate à pobreza que levem em consideração as particularidades e vocações locais, conforme se propõe a metodologia para a produção do conhecimento socialmente robusto (MPCSR), formulada por Ritto (2010). A MPCSR configura-se em medidas propositivas para geração de trabalho e renda e protagonismo social, o que aumenta a possibilidade de promover maior independência dos beneficiários dos programas de redistribuição de renda, entre outras políticas públicas de combate à pobreza na esfera federal, no médio e no longo prazo, contribuindo para a possível redução da dependência dos programas pelos beneficiários e sua perpetuação na condição de pessoa pobre, e por fim reduzindo-se, portanto, o risco de interrupção dos benefícios em momentos de crise e instabilidade política e econômica. Nesse aspecto, a MPCSR propõe ações articuladas por meio de projetos que vinculem o poder público e o privado que se utilizem das potencialidades do local, bem como a cultura, as vocações locais (identificadas ou não) para o desenvolvimento de trabalho e renda, reconhecendo e valorizando as diferenças, permitindo o deslocamento do que é considerado inferior para o nível equivalente ao nível superior no paradigma da monocultura da naturalização das diferenças e das ecologias dos saberes, formulado por Boaventura (Motta, 2012) e, por conseguinte, possível mobilidade social.

Por fim, cumpre ressaltar que intervenções focalizadas nos pobres e com objetivo de alívio imediato de privações demandam a ruptura do paradigma do critério de elegibilidade unidimensional para critério multidimensional que privilegie pessoas em condições de pobreza em todos os ciclos da vida e que consistiam para além da promoção de serviços básicos essenciais, mas que assegurem a todas e todos e a sua família saúde e bem-estar e segurança, assim como preconiza a Declaração Universal dos Direitos Humanos.

\section{PROPOSTA PARA ESTUDOS POSTERIORES}

Considerando que a pesquisa exploratória tem por finalidade a formulação de problemas mais precisos e, sobretudo, proporcionar visão geral acerca de determinado fato para estudos posteriores, propomos a realização de estudos científicos para subsidiar a formulação de políticas públicas que incluam o contingente residual de pessoas não assistidas pelos programas cujo critério de elegibilidade seja o unidimensional e que, portanto, não levam em consideração outros tipos de privações, para além do determinante econômico, as quais limitam o bem-estar social dessas famílias.

Por fim, diante do limite desta pesquisa, acerca da impossibilidade de análise da mobilidade positiva de todas as 23 variáveis na vida dos membros da geração G3, crianças e jovens, é que propomos, no âmbito do complexo de favelas do São João, a realização de pesquisa comparativa, adotando a estratégica metodológica aplicada neste estudo, incluindo os membros das gerações G4 e G5 das famílias que participaram desta pesquisa, bem como outros grupos de moradores que não fizeram parte, para análise do possível rompimento ou não do ciclo da pobreza entre 
as gerações de grupos beneficiários de programas sociais e não beneficiários de programas de combate à pobreza implantados nos últimos dez anos.

\section{NOTAS}

1. Este artigo incorpora e complementa parte do trabalho apresentado no $7^{\circ}$ Congresso Ibero-Americano em Investigação Qualitativa (CIAIQ2018), 2018, intitulado: "Reprodução intergeracional da pobreza: o caso do complexo de favelas do São João - Rio de Janeiro - Brasil".

2. Em termos normativos, a meta de erradicação da pobreza está ancorada na abordagem dos direitos humanos. Isso pode ser visto em diferentes instrumentos assinados pelos países no marco das Nações Unidas, como a Declaração Universal de Direitos Humanos (1948), o Pacto Internacional de Direitos Econômicos, Sociais e Culturais (1966), a Reunião de Cúpula Mundial de Desenvolvimento Social (1995) e a Reunião de Cúpula do Desenvolvimento do Milênio (2000). Com base nesse enfoque, os pobres são titulares de direitos que devem ser garantidos pelos Estados, e a erradicação da pobreza é essencial para o respeito da dignidade humana de todas as pessoas (Cadernos de Estudos Desenvolvimento Social em Debate, no 15 (2013). Brasília: Ministério do Desenvolvimento Social e Combate à Fome/Secretaria de Avaliação e Gestão da Informação, 2005. 88 p. II. Feres, Juan Carlos. III. Villatoro, Pablo).

3. Termo cunhado pelo sociólogo brasileiro Wanderley Guilherme dos Santos para designar uma cidadania restrita aos trabalhadores formais. (Santos, 1994).

4. Pobreza transitória apresenta uma natureza temporária. Entre os fatores que contribuem para a pobreza transitória estão: tamanho da família, transferências governamentais de renda, sazonalidade de atividades econômicas, migração e eventos de ciclo de vida, segundo McKay e Lawson (2002).

5. Mobilidade social diz respeito à transição de uma posição social a outra realizada por um indivíduo (Johnson, 1997).

6. Pobreza crônica: condições de privação que um indivíduo experimenta ao longo de sua vida (Hulme; Shepherd, 2003; 2005).

7. Unidade de polícia pacificadora (UPP): de acordo com o previsto no Decreto $n^{\circ} 45.186$, de 17 de março de 2015, o Programa de Polícia Pacificadora tem como objetivos a recuperação de territórios sob o controle de grupos ilegais armados, a restauração do monopólio legal e legítimo da força pelo Estado e a diminuição da criminalidade violenta, sobretudo a letal. Fonte: http://www.isp.rj.gov.br/Conteudo. asp?ident=62. Acesso em: 05 jan. 2018. 


\section{REFERÊNCIAS}

Barros, Ricardo Paes; Carvalho, Mirela; Franco, Samuel (2006). Pobreza multidimensional no Brasil. Rio de Janeiro: IPEA. (Textos para discussão n 1.227).

Bird, K. (2007). The intergenerational transmission of poverty: an overview. Chronic Poverty Research Centre Working Paper no 99. Doi: http://dx.doi.org/10.2139/ssrn.1629262.

Bird, K. (2010). Using life history research as part of a mixed methods strategy to explore resilience in conflict and post-conflict settings. CPRC Conference Paper. September. Ten years of 'war against poverty' what have we learned since 2000 and what should we do 2010-2020.

Bourdieu, Pierre. A escola conservadora. In: ; Nogueira, M. A.; Catani, A. (Orgs.) (1998). Escritos de educação (p. 39-64). Petrópolis: Vozes.

Cain, E. (2009).Social protection and vulnerability, risk and exclusion across the life-cycle. In: OECD. Promoting pro.poor growth: employment and social protection. Paris: OECD. Disponível em: www. oecd.org/dataoecd/63/10/43514563.pdf.

Crespo, Antônio Pedro Albernaz; Gurovitz, Elaine (2002). Administração pública - a pobreza como fenômeno multidimensional. RAE-eletrônica, 1, n. 2, 11, jul./dez. Doi: http://dx.doi.org/10.1590/ S1676-56482002000200003.

Doeringer, P.; Piore, M. (1971). Internal labor market and manpower analysis. Lexington: Heath Lexington Books.

Draibe, Sônia (2003). A política social no período FHC e o sistema de proteção social. Tempo Social, São Paulo, v. 15 n. 2, nov. Disponível em: http://dx.doi.org/10.1590/S0103-20702003000200004. Acesso em: 10 jan. 2018.

Esteves, António J. (1986). A investigação-acção. In: Pinto, J. M; Silva. A. S. Metodologia das Ciências Sociais. (pp. 251-278). Porto: Afrontamento.

Feres, Juan Carlos; Villatoro, Pablo (2013). Cadernos de estudos Desenvolvimento Social em Debate, n. 15. Brasília: Ministério do Desenvolvimento Social e Combate à Fome; Secretaria de Avaliação e Gestão da Informação.

Guerra, Isabel Carvalho (2006). Pesquisa qualitativa e análise de conteúdo - sentidos e forma de uso. Estoril: Principia.

Hagenaars, Aldi; De Vos, Klaas (1988). The definition and measurement of poverty. The Journal of Human Resources, 23, n. 2, 211-221, spring.

Hulme, D.; Shepherd, A. (2003). Conceptualizing Chronic Poverty. World Development, 31(3), 403-423.

Hulme, D.; Shepherd, A. (2005). Identifying and understanding chronic poverty: beyond monetary measures. Manchester: University of Manchester and Chronic Poverty Research Centre.

Johnson, A. G. (1997). Dicionário de Sociologia. Guia prático de linguagem sociológica. Rio de Janeiro: Jorge Zahar. 
McKay, A.; Lawson, D. (2002). Chronic poverty: a review of current quantitative evidence. Chronic Poverty Research Centre (CPRC). Working Paper 15.

Minayo, M. C. S. (2007). Análise qualitativa: teoria, passos e fidedignidade. Ciências \& Saúde Coletiva, 17(3), 621-626.

Motta, A. M. A produção do conhecimento socialmente robusto no contexto da educação e do trabalho: inserção de moradores em comunidades do Rio de Janeiro no mercado de trabalho formal. 2012. 165 f. Dissertação (Mestrado em Políticas Públicas e Formação Humana) - Faculdade de Educação, Universidade do Estado do Rio de Janeiro.

Motta, A. M.; Parente, C. Reprodução intergeracional da pobreza: o caso do complexo de favelas do São João - Rio de Janeiro - Brasil. $7^{\circ}$ Congresso Ibero-Americano em Investigação Qualitativa (CIAIQ2018). Fortaleza/CE, 2018.

Raczynski, Dagmar (2002). Equidad, inversión social y pobreza. Innovaren como se concibe, diseña y gestionala politicas y los programas sociales. Mimeo. Documento preparado para o Seminario Perspectivas Innovativas en Política Social. Desigualdades y Reducción de Brechas de Equidad, Mideplan - Cepal, 23-24 mayo.

Ritto, A. (2010). Metodologia para produção de conhecimento socialmente robusto. Rio de Janeiro: Ciência Moderna.

Serapioni, Mauro (2016). Conceitos e métodos para a avaliação de programas sociais e políticas públicas. Sociologia, Revista da Faculdade de Letras da Universidade do Porto, XXXI, 59-80.

Santos, Wanderley Guilherme dos (1994). Cidadania e Justiça: a política social na ordem brasileira. $3^{\mathrm{a}}$ ed. Rio de Janeiro: Campus.

Sen, Amartya (2000). Desenvolvimento como liberdade. São Paulo: Companhia das Letras.

Yazbek, Maria Carmelita (2012). Pobreza no Brasil contemporâneo e formas de seu enfrentamento. Serv. Soc., 110, p. 288-322, abr./jun. Disponível em: http://www.cressrn.org.br/files/arquivos/ j01k8G2OYc356230ru47.pdf 